\title{
Exercise programs for LVAD supported patients: A snapshot from the ESC affiliated countries
}

Tuvia Ben Gal, Massimo F. Piepoli, Ugo Corra, Viviane Conraads, Stamatis Adamopoulos, Piergiuseppe Agostoni, Ewa Piotrowicz, Jean-Paul Schmid, Petar M. Seferovic, Piotr Ponikowski, Gerasimos Filippatos and Tiny Jaarsma

\section{Linköping University Post Print}

\section{Tweet}

N.B.: When citing this work, cite the original article.

Original Publication:

Tuvia Ben Gal, Massimo F. Piepoli, Ugo Corra, Viviane Conraads, Stamatis Adamopoulos, Piergiuseppe Agostoni, Ewa Piotrowicz, Jean-Paul Schmid, Petar M. Seferovic, Piotr Ponikowski, Gerasimos Filippatos and Tiny Jaarsma, Exercise programs for LVAD supported patients: A snapshot from the ESC affiliated countries, 2015, International Journal of Cardiology, (201), 215-219.

http://dx.doi.org/10.1016/j.ijcard.2015.08.081

Copyright: Elsevier

$$
\text { http://www.elsevier.com/ }
$$

Postprint available at: Linköping University Electronic Press

http://urn.kb.se/resolve?urn=urn:nbn:se:liu:diva-123748

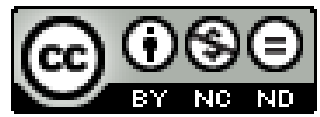


Exercise programs for LVAD supported patients: a snapshot from the ESC affiliated countries.

Tuvia Ben Gal ${ }^{1}$, Massimo F Piepoli ${ }^{2}$, Ugo Corrà ${ }^{3}$, Viviane Conraads ${ }^{4}$, Stamatis Adamopoulos ${ }^{5}$, Piergiuseppe Agostoni ${ }^{6}$, Ewa Piotrowicz ${ }^{7}$, Jean-Paul Schmid ${ }^{8}$, Petar M. Seferovic ${ }^{9}$, Piotr Ponikowski $^{10}$, Gerasimos Filippatos ${ }^{11}$, Tiny Jaarsma ${ }^{12}$. on behalf of the Committee on Exercise Physiology \& Training of the Heart Failure Association and endorsed by the Cardiac Rehabilitation Section of the European Association for Cardiovascular Rehabilitation and Prevention of the ESC.

1. Heart Failure Unit, Rabin Medical Center, Petah Tikva, Israel. Affiliated to the Sackler Faculty of Medicine, Tel Aviv University, Tel Aviv, Israel

2. Heart Failure Unit, Cardiac Dept, G. da Saliceto Polichirurgico Hospital, Piacenza, Italy

3. Department of Cardiology, Fondazione Salvatore Maugeri, IRCCS Scientific Institute of Veruno, Veruno, Italy

4. Department of Cardiology and Cardiac Rehabilitation, Antwerp University Hospital, Edegem, Belgium

5. Department of Cardiology, Onassis Cardiac Surgery Center, Athens, Greece

6. Centro Cardiologico Monzino, IRCCS, Milano, Italy

7. Telecardiology Center, Institute of Cardiology, Alpejska 42, 04-628, Warszawa, Poland

8. Cardiology Clinic, Tiefenau Hospital and University of Bern, Bern, Switzerland

9. Department of Cardiology, Clinical Center of Serbia, University of Belgrade School of Medicine, Belgrade, Serbia. Serbian Academy of Sciences and Arts

10. Department of Heart Diseases, Wroclaw Medical University, Wroclaw, Poland 
11. Heart Failure Unit at the Department of Cardiology, Athens University Hospital Attikon, Greece

12. Department of Social and Welfare Studies, University of Linkoping, Sweden

Address for correspondence

Tuvia Ben Gal, MD.

Head of the Heart Failure Unit

Director of the Heart Transplantation and LVAD Clinics

Cardiology Department, Rabin Medical Center

Petah Tikva, Israel

Tel: +972522757616

Fax: +97239377337

E-mail: bengaltu@gmail.com

Key words: Cardiac rehabilitation, exercise training, heart failure, Left ventricular assist device. 


\section{ABSTRACT:}

Background: To contribute to the protocol development of exercise training in LVAD supported patients by reviewing the exercise programs for those patients in the ESC affiliated countries. Methods: A subset of data from 77 (26 countries) LVAD implanting centers that participated in the Extra-HF survey (170 centers) was analyzed.

Results: Of the 77 LVAD implanting centers, 45 (58\%) reported to have a functioning exercise training program (ETP) for LVAD patients. In 21 (47\%) of the 45 ETP programs in LVAD implanting centers, patients begin their ETP during their in-hospital post-operative recovery period. Most centers (71\%) have an early post-discharge program for their patients, and 24\% of the centers offer a long-term maintenance program. The professionals involved in the ETPs are mainly physiotherapists (73\%), psychologists, cardiac rehab nurses (22\%), or cardiologists specialized in rehabilitation (22\%). Not all programs include the treating cardiologist or surgeons. Most of the ETPs (84\%) include aerobic endurance training, mostly cycling (73\%), or walking (62\%) at low intensity intervals. Some programs apply resistance training (47\%), respiratory muscle training (55\%), or balance training (44\%).

Reasons for the absence of ETPs are referral of patients to another center (14 centers) and lack of resources (11 centers).

Conclusion: There is a great variance in ETPs in LVAD implanting centers.. Not all the implanting centers have an ETP, and those that do have adopted a local protocol.. Clear guidance on ETP supplied by LVAD implanting centers to LVAD supported patients and more evidence for optimal modalities is needed. (249 words) 


\section{INTRODUCTION:}

With the advances in the mechanical circulatory support technologies, a growing group of patients with end- stage heart failure (HF) who do not have any other therapeutic option, can now benefit from implantation of a left ventricular assist device (LVAD). Indications for LVAD implantation include: bridge to heart transplantation, bridge to transplant ability, bridge to recovery, and recently also as destination (permanent) therapy [1].

Support by an LVAD generally leads to improvement in functional capacity, quality of life, and survival in patients with advanced HF [2] however cost effectiveness is still discussed [3] . Research on exercise in patients after an assist device implantation is still scarce and under development, but the few studies that have addressed cardiac rehabilitation of these recipients have found exercise rehabilitation after device implantation to be beneficial for improving patients' recovery [4-8]. Although clear clinical guidelines, established exercising protocols and hard evidence on the effectiveness of exercise in LVAD patients are lacking, there is a general opinion that physical training is safe and beneficial for patients supported with an LVAD [9]. Preliminary data also show benefits of exercise training in the native heart itself of trained LVAD supported patients [10]. In a recently published systematic review, 6 studies describing physical therapy or exercise in LVAD patients were found [11]. The authors concluded that cardiac rehabilitation is safe for these patients as early as two weeks post implantation, based upon the patient's status as defined by each individual study. However, they also concluded that 'the available evidence is not strong enough to draw conclusive clinical guidelines for patients status post LVAD placement in acute care or rehabilitation settings [11].

Lack of evidence- based guidelines leads to uncertainty on the optimal timing, duration and intensity of the training protocol for rehabilitation of LVAD supported patients. Therefore, a clear 
protocol that can be tested, adapted over time, and that is based on current practice and experience might improve practice. To contribute to the protocol development, we aimed to describe the content of exercise programs (ETP) for LVAD supported patients in the member countries of the Heart failure Association of the European Society of Cardiology (ESC).

\section{METHOD}

For this paper, a subset of data from the Extra-HF survey was analyzed. The Extra-HF survey was an initiative of the Heart Failure Association of the ESC and consisted of a survey performed in countries that are affiliated to the ESC. The results of the main Extra-HF survey have been described (12) and the current paper reports unique data from the LVAD implanting centers and the ETP provided to LVAD patients.

\section{Instrument:}

The Extra-HF Survey-Questionnaire: The questionnaire was developed and refined by the Committee members of the Exercise Physiology \& Training of the Heart Failure Association (HFA). The survey was translated into a web-form [http://www.formstack.com/forms/escardiohfa_survey] and managed by a staff member of the European Heart House. A part of this questionnaire was specifically dedicated to rehabilitation of LVAD patients and included sections on the characteristics of the reference hospital/health centers, dimension of the treated LVAD population, and underlying factors for not having an ETP. Where ETP for LVAD patients was 
available, there were questions on coverage of costs, involved staff, the modality of exercise training (aerobic, resistance, balance, respiratory), and the tests performed to evaluate the efficacy of the ETP in LVAD patients.

Within the questionnaire, we differentiated between three phases of ETP, defined as:

- Phase I: in-hospital program.

- Phase II: early post-discharge program. This period is usually defined as the first 2-16 weeks after discharge, when structured and closely monitored exercise, psycho-educational activities and lifestyle changes are encouraged.

- Phase III: long-term maintenance program. This is the continuation period that usually involves less intense supervision.

\section{Procedure}

Identification and approach to national coordinators: The first step in the data collection was to contact the chairs of the national heart failure societies and/or working groups in the ESC member's countries. For countries without national HF working groups, we used a number of approaches, including informal contact by committee members of the HFA, and/or writing to the national societies belonging to the ESC. Initially, 46 national HF coordinators were identified. They were sent tie link to the questionnaire, asking them to forward the surveys to the relevant professionals in their countries. The survey was launched after the May 2013 HFA Scientific meeting in Lisbon, and data was collected until February 2014.

Analysis: Descriptive statistics were performed. For purposes of readability, the response categories 'always/ mostly/ usually' were collapsed and reported as 'yes', and the categories 
'seldom/not usually/not' were collapsed as 'not'. In some questions, there could be more than one answer to the same question, which is why the percentages reported exceed $100 \%$.

\section{RESULTS}

\section{$\underline{\text { Respondents }}$}

The Extra-HF survey included data from 170 centers, of which 77 (from 26 countries) reported to be an LVAD implanting center. The 77 responding LVAD implanting centers were mostly universities ( $\mathrm{n}=49,64 \%)$, or general hospitals ( $\mathrm{n}=11,14 \%)$, with mainly public funding $(\mathrm{n}=61$, 79\%). Of the 77 LVAD centers, 45 centers (58\%) reported to have a functioning ETP for LVAD patients. In the 77 implanting centers the number of implants in the last calendar year was reported to vary between 1 and 300, with a median of 10 per year.

\section{General follow- up of LVAD patients}

In the 45 centers with an ETP, the general follow- up of the LVAD implanted patients was performed by the cardiology department $(8,18 \%)$, the cardiac surgery department $(18,40 \%)$, or the HF unit (19, 42\%). In a minority of the responding centers (5, 11\%), the general follow- up of LVAD implanted patients was performed in different combinations: HF and heart transplant units, or main department (cardiology or cardiac surgery) and general hospital clinics. In the centers without LVAD_ETP $(n=32), 19(60 \%)$ had a low number of LVAD implants a year $(<10)$ but $40 \%$ of these centers had 10 or more LVAD implants a year. In the centers with an LVAD ETP (n=45), 49\% have a low number of LVAD implants $(<10)$ and 51\% have 10 or more LVAD implants a year. 


\section{Organization of the Exercise Training Program in the LVAD centers (Table 1)}

ETP time frames: In 21 centers, LVAD implanted patients started their exercise training during their in-hospital post-operative recovery period, as early as 2 weeks after the LVAD implantation. Most centers (71\%) offer an ETP for the early post discharge period, defined as from 2 weeks post discharge. The duration of the early post discharge ETP was mostly 4 weeks in 22 (49\%) centers with in-hospital setting, 4-12 weeks in 13 (29\%) centers with an out-patient setting and up to a year in 4 (9\%) centers with home based ETP. Only 11 (24\%) LVAD implanting centers had a long- term in-house ETP for their LVAD implanted patients, with a wide range in duration (2-52 weeks).

Professionals involved in the ETP: In general, there was a large variety in the composition of the rehabilitation team. Most rehabilitation teams included a physiotherapist (33, 73\%), a nurse (either cardiac rehabilitation nurse or HF nurse), a rehabilitation specialist (e.g., a cardiologist specialized in rehabilitation (22, 49\%), an exercise physiologist or therapist $(16,36 \%)$, and a specialist in rehabilitation $(10,22 \%))$. The treating physicians were only rarely involved in their LVAD implanted patients’ ETP: the cardiologist was involved in only 17 (38\%) of the ETP's and the cardiac surgeon in $10(22 \%)$,

Number of patients per session: The number of LVAD patients trained in groups was low: most centers $(25,61 \%)$ reported to have individual sessions, while 7 centers (17\%) trained two patients, and 6 centers (15\%) three patients per session. Monitoring and follow- up: Most centers (53\%) used ECG and/or blood pressure (49\%) to monitor their patients during exercise. In only 22 centers, the device parameters were adapted according to the performance of the patients. Tests routinely performed included echocardiography 
(36, 80\%), 6 minute walk test (6MWT) (30, 67\%), quality of life assessment (28, 40\%), and bicycle cardiopulmonary exercise test (24, 53\%).

Content of the training program in LVAD centers. (Table 2)

Most of the exercise training protocols (84\%) included aerobic endurance training on a bicycle (73\%), or walking on a treadmill (62\%) at low intensity intervals. Resistance training was applied in $49 \%$ of the programs, using exercise equipment like resistance bands, free weights, exercise balls and so on. Some programs applied resistance training (47\%), respiratory muscle training (55\%), or balance training (44\%).

$\underline{\text { Reasons for lack of ETP: }}$

The reasons for the absence of an ETP were diverse and multifactorial among the $32 \mathrm{LVAD}$ implanting centers without a program (Table 3). Several (44\%) indicated that they referred their LVAD implanted patients to another cardiac rehabilitation program in the area. Lack of resources was one of the main hurdles for the incorporation of ETP into LVAD programs, with most centers reporting financial or logistic problems, such as transportation or accommodation. In 11 (34\%) of the LVAD implanting centers without an ETP, such a program was not reimbursed by the referring institution or the local health care system. Lack of a specialized team, resulting in concerns about having the right skills and knowledge on how to manage LVAD patients, was the reason for not having an ETP for LVAD patients in 6 (19\%) centers.

\section{DISCUSSION}


This is a first snapshot on the prevalence, content, and protocols of exercise training programs for LVAD supported patients in the ESC member countries. The first impression is that there is a great variety in the availability of ETP in the LVAD centers, as well as in intensity and format of the programs, methods of monitoring and follow-up. Such a variety can be explained partly by the fact that it has only been a decade since re-designed axial flow LVADs, showed to be safe and effective, became a regular treatment for patients with end-stage HF [13]. During these years, only a few studies addressed cardiac rehabilitation of LVAD recipients. Thus, the optimal timing, duration and intensity, or in other words, an evidence- based training protocol for the rehabilitation of LVAD implanted patients, considering the peculiarities of this "new" group of cardiovascular patients, have not yet been clearly described [6,14]. Although based on limited evidence [4,14], intuitively extrapolating the beneficial effects of rehabilitation of patients recovering from other cardiac events like acute coronary events, heart transplantation, or cardiac surgery, it is suggested that in general, exercise rehabilitation after LVAD implantation should have positive effects on LVAD supported patients' recovery. A unique difficulty faced by LVAD supported patients early post- implantation is the need to embrace the batteries and controller to become a part of their body. The patients must adapt to the physical and psychological burden of carrying the two batteries and the controller weighing approximately 2-2.5 kilograms [14]. As presented in our study, the lack of clear guidelines for the requirements from an LVAD implanting center and for the proper management of LVAD implanted patients, as well as the differences in the health care systems in the different ESC countries, resulted in the diverse characteristics of the various LVAD programs and in the absence of ETP in as many as $42 \%$ of the LVAD implanting centers. The intuitive usefulness of rehabilitation in LVAD supported 
patients is reflected in the referral of patients from $44 \%$ of the implanting centers with no EPT to another rehabilitation center.

Although multifactorial, the main hurdle for the incorporation of ETP in the LVAD program is lack of resources, but not because of safety concerns. Training and financing appropriate and specialized staff might be expensive mainly in terms of money, but also in terms of time. Proper reimbursement is crucial for financing the LVAD programs in general and ETP for LVAD implanted patients in particular. The lack of reimbursement can partly be explained by the lack of evidence on the benefits expected from the ETP in LVAD implanted patients. Most of the training equipment must have the ability to start at a very low load and strengthen the upper girdle (for carrying the batteries' bag), without causing excessive stress to the just operated sternum. Some centers reported that they could not afford to purchase the appropriate equipment. In order to achieve excellence and good practice, in most countries, only the large medical centers, usually those that perform heart transplantation, implant LVADs in patients referred from distant places. The LVAD implanted patients living far away from the center where they had their LVAD implanted find it difficult to participate in the ETP performed there. Other LVAD implanting centers are located far away from densely populated regions, making it difficult, but feasible, for the patients to attend their scheduled follow- up visits. However, it is completely unpractical to participate in a regular ETP twice a week.

In the absence of a dedicated ETP for LVAD recipients or in the case of limited number of such patients in centers that value its benefits but cannot afford it, incorporating the LVAD recipients in the general cardiology (or dedicated to HF) ETP might be an acceptable solution if implementation of the appropriate training protocol, which remains to be defined, is performed. 
In a recent study on a limited number of patients [14], a well-constructed exercise training program with well-defined training protocol performed on patients supported with a LVAD in the very early post-operative, pre-discharge period was found to be safe and led to improved functional capacity. The scarcity of data on the appropriate timing of initiating the ETP for LVAD recipients leads to very diverse protocols, both in terms of initiation and duration. Among the centers responding to our survey, $47 \%$ have an in-hospital ETP (phase I), and $71 \%$ have an early post -discharge ETP for LVAD recipients (phase II). The duration of the ETP varies between 4-12 weeks (mostly 4 weeks), and only a minority of the programs are long term. Only $21 \%$ of the implanting centers have a long- term ETP (phase III).

Another interesting point of this survey was the variety in staffing of ETP programs for LVAD patients. Although the core components, standards, and outcome measures for referral and delivery, as well as the professionals involved in a general cardiac rehabilitation program seem to be rather well defined [15], the characteristics regarding the training required, and the professions of those allowed to run an ETP for LVAD recipients have not yet been determined. There is a lack of definition of the knowledge and skills that are needed to provide optimal rehabilitation of these patients. Organizations involved in the training of professionals might accept this challenge and design a training program for those who are to be involved in ETP programs for LVAD patients.

With regard to the content and organization of the ETP programs, we found that most centers reported a very low number of patients participating in each training session. The reason is probably the low volume of LVAD implants performed at each center. Training in larger groups has some benefits, mainly social and psychological [16], which are missed in individual training On the other hand, it has to be considered that small group training might be justified since these 12 
patients may require increased attention for safety reasons (carrying batteries, cables etc.). Therefore, other options for providing social and psychological support for LVAD patients might be needed, such as post- discharge group sessions or maybe use of social media.

There are different exercise-training protocols aiming at specific populations, both healthy and diseased. Most of these protocols combine aerobic and anaerobic, as well as endurance and resistance training. Most LVAD implanting centers applied aerobic endurance training at intervals of low intensity for their LVAD patients, such as biking. Approximately half of the patients performed resistance training using free weights and other equipment and more than half trained their respiratory muscles. Not even one center reported on special measures taken to deal with the peculiarity of performing the exercise training with a bag weighing 2.3 kilograms carried either on the patients back or held at a very close distance from the training patient. This variety in the content of exercise training for LVAD recipients reflects the non-homogeneity of the exercising programs that results from the absence of a suggested protocol. We found that centers with a larger number of patients being implanted have more often a ETP for LVAD patients then the centers with a lower volume $(<10)$. However, we also found that the volume is not the main explanation for having or not having a ETP for VAD patients. On one hand some sites with a low number $(<10)$ of implants a year do actually have an ETP for their LVAD patients and on the other hand half of the larger sites (10 or more) did not have an ETP for their LVAD patients. The individual training program depends on the physical condition of every exercising patient, but no matter what protocol is used, the patient's condition should be assessed periodically and the individualized exercising program adapted accordingly. With regard to the assessment during the ETP, there seemed to be more uniformity in the centers in our survey. In most centers, patients performed the 6MWT, the bicycle cardiopulmonary exercise test, and quality of life was 
assessed. Although the 6MWT is a useful, reliable, informative and easy test to perform among LVAD supported patients [17], cardiopulmonary exercise testing, usually by using a bicycle ergometer, is more informative, but more complicated to perform [18]. The fact that more than $50 \%$ of the centers use this test reflects the high quality of the training center. During exercise, compensation for the need for increased cardiac output was addressed by the manufacturing companies, with an attempt to change the device parameters according to the exercise intensity. It is yet unknown whether while exercising, the device parameters can be maintained as at rest or whether they need to be adjusted (speed increased) during physical activity. It can be assumed with a high degree of certainty that as the cardiac output generated by the device is afterload sensitive [19], the peripheral vasodilatation during exercise probably results in an increase in the effective cardiac output generated by the device. Thus, although some centers did change the device parameters while exercising, this was probably not needed. Another questionable issue concerns the best way to monitor the exercising LVAD recipient. Monitoring of patients supported by an LVAD during exercise training is complicated by the continuous flow hemodynamics, resulting in absence or weakening of the peripheral pulse. ECG monitoring, used in $53 \%$ of the centers, is easy to apply, but its significance is unclear. Furthermore, the blood pressure measured by $49 \%$ of the centers needs further elaboration because the cuff measurements are not reliable. Post- exercise blood pressure measured with a Doppler probe may improve the general therapeutic approach to the patient by adapting the appropriate medical therapy.

We realize that our study is a survey study, limited by the partial response of the LVAD implanting centers. However, by using the national coordinators of the HFA, we assume that the 
responses we did receive came from the large and influential centers. We invite other centers to complete our information database if they feel they have been excluded.

In summary, this study describes the clear lack of uniformity in ETPs in the LVAD implanting centers. Not all the implanting centers have an ETP, and those that do have adopted a local exercise training protocol. Almost half of the centers that do not have an ETP refer their patients to other rehabilitation programs, implying that across Europe, ETP is performed even in high risk patients. The benefits of exercise training in LVAD recipients, though intuitively expected, need to be futher studied by well-designed studies. Additional studies are needed into the role of alternative modalities of exercise training such as for example using electoral muscle stimulation that has been shown to be an effective alternative modality in HF patients or the role of other novel forms of exercise such as exergaming (When proven, every LVAD implanting center will have to present an ETP to be accredited as an LVAD implanting center. The personnel, equipment, training protocol, as well as the suggested timing of initiation and duration should be clearly defined. The impact of ETP on the survival of LVAD supported patients needs to be studied as well. 
Acknowledgments:

1. This work is dedicated to Viviane Conraads who passed away at the age of 50, during the process of finalizing this project. She is greatly missed by all who had the privilege to know her.

2. We would like to thank the Heart failure Association of the ESC for the financial support of this survey.

3. Finally, we wish to thank all the National Coordinators in the different European Countries.

4. There are no financial or other relations that could lead to a conflict of interest. 


\section{REFERENCES:}

1. Mancini D, Colomb P. Left ventricular assist devices. A Rapidly Evolving Alternative to Transplant. J Am Coll Cardiol 2015;65:2542-55.

2. Starling RC, Naka Y, Boyle AJ, Gonzalez-Stawinski G, John R, Jorde U, Russell SD, Conte JV, Aaronson KD, McGee EC Jr, Cotts WG, DeNofrio D, Pham DT, Farrar DJ, Pagani FD. Results of the post-U.S. Food and Drug Administration-approval study with a continuous flow left ventricular assist device as a bridge to heart transplantation: a prospective study using the INTERMACS (Interagency Registry for Mechanically Assisted Circulatory Support). J Am Coll Cardiol 2011;57(19):1890-8.

3. Clarke A, Pulikottil-Jacob R, Connock M, Suri G, Kandala NB, Maheswaran H, Banner NR, Sutcliffe P Cost-effectiveness of left ventricular assist devices (LVADs) for patients with advanced heart failure: analysis of the British NHS bridge to transplant (BTT) program. Int J Cardiol. 2014;171(3):338-45.

4. Laoutaris ID, Dritsas A, Adamopoulos S, Manginas A, Gouziouta A, Kallistratos MS, Koulopoulou M, Voudris V, Cokkinos DV, Sfirakis P. Benefits of physical training on exercise capacity, inspiratory muscle function, and quality of life in patients with ventricular assist devices long-term post implantation. Eur J Cardiovasc Prev Rehab 2011;18(1):33-40. 
5. Ueno A, Tomizawa Y. Cardiac rehabilitation and artificial heart devices. Journal of Artif Organs 2009;12(2):90-7.

6. Hayes K, Leet AS, Bradley SJ, Holland AE. Effects of exercise training on exercise capacity and quality of life in patients with a left ventricular assist device: a preliminary randomized controlled trial. J Heart Lung Transpl. 2012;31(7):729-34.

7. Bellotto F, Compostella L, Agostoni P, Torregrossa G, Setzu T, Gambino A, Russo N, Feltrin G, Tarzia V, Gerosa G. Peripheral adaptation mechanisms in physical training and cardiac rehabilitation: the case of a patient supported by a CardioWest total artificial heart. J Card Fail. 2011;17(8):670-5.

8. Christle JW, Boscheri A, Pressler A, Grinninger C, Schramm R, Hagl CM, Halle M. Interval exercise training increases maximal and submaximal exercise performance in heart failure with biventricular assist device therapy.Int J Cardiol. 2015 May 6;187:104-5.

9. Alsara O, Perez-Terzic C, Squires RW, Dandamudi S, Miranda WR, Park SJ, Thomas RJ. Is exercise training safe and beneficial in patients receiving left ventricular assist device therapy? J Cardiopulm Rehabil Prev 2014;;34(4):233-40.

10. Adamopoulos S, Gouziouta A, Mantzouratou P, Laoutaris ID, Dritsas A, Cokkinos DV, Mourouzis I, Sfyrakis P, Iervasi G, Pantos C. Thyroid hormone signaling is altered in response to physical training in patients with end-stage heart failure and mechanical assist devices: potential physiological consequences? Interactive CardioVascular and Thoracic Surgery; 2013:17 (4):664-8.

11. Scheiderer R, Belden C, Schwab D, Haney C, Paz J. Exercise guidelines for inpatients following ventricular assist device placement: a systematic review of the literature. Cardiopulm Phys Ther J 2013;24(2):35-42. 
12. Piepoli MF, Binno S, Corrà U, Seferovic P, Conraads V, Jaarsma T, Schmid JP, Ponikowski PP on behalf of the Committee on Exercise Physiology \& Training of the Heart Failure Association and endorsed by the Cardiac Rehabilitation Section of the European Association for Cardiovascular Rehabilitation and Prevention of the ESC. European exercise training in heart failure survey. Eur J Heart Fail 2015;17(6):631-638 .

13. Frazier OH1, Delgado RM 3rd, Kar B, Patel V, Gregoric ID, Myers TJ First clinical use of the redesigned HeartMate II left ventricular assist system in the United States: a case report. Tex Heart Inst J 2004;31(2):157-9.

14. Blumberg Y, Kravits A, Dinkin D, Neimark A, Abu-Hatzira M, Shtein R, Yaari V, Medalion B, Battler A, Pinchas A, Ben Gal T. Early Physical Rehabilitation after Axial Flow Left Ventricular Assist Device Implantation: suggested protocol and a pilot study. Int J Phys Med \& Rehab 3;2 (1000263): 2015. doi.org/10.4172/2329-9096.1000263.

15. Piepoli MF, Corrà U, Adamopoulos S, Benzer W, Bjarnason-Wehrens B, Cupples M, Dendale P, Doherty P, Gaita D, Höfer S, McGee H, Mendes M, Niebauer J, Pogosova N, Garcia-Porrero E, Rauch B, Schmid JP, Giannuzzi P. Secondary prevention in the clinical management of patients with cardiovascular diseases. Core components, standards and outcome measures for referral and delivery: A Policy Statement from the Cardiac Rehabilitation Section of the European Association for Cardiovascular Prevention \& Rehabilitation. Endorsed by the Committee for Practice Guidelines of the European Society of Cardiology. Eur J Prev Cardiol 2012;21(6):664-81.

16. Hui E, Yang H, Chan LS, Or K, Lee DT, Yu CM, Woo J. A community model of group rehabilitation for older patients with chronic heart failure: a pilot study. Disabil Rehabil 2006;28(23):1491-7. 
17. Hasin T, Topilsky Y, Kremers WK, Boilson BA, Schirger JA, Edwards BS, Clavell AL, Rodeheffer RJ, Frantz RP, Joyce L, Daly R, Stulak JM, Kushwaha SS, Park SJ, Pereira NL. Usefulness of the six-minute walk test after continuous axial flow left ventricular device implantation to predict survival. Am J Cardiol 2012;110(9):1322-8.

18. Dunlay SM, Allison TG, Pereira NL. Changes in cardiopulmonary exercise testing parameters following continuous flow left ventricular assist device implantation and heart transplantation. J Card Fail 2014;20(8):548-54.

19. Lampert BC, Eckert C, Weaver S, Scanlon A, Lockard K, Allen C, Kunz N, Bermudez C, Bhama JK, Shullo MA, Kormos RL, Dew MA, Teuteberg JJ. Blood pressure control in continuous flow left ventricular assist devices: efficacy and impact on adverse events. Ann Thorac Surg 2014;97(1):139-46.

20. Sbruzzi G, Ribeiro RA, Schaan BD, Signori LU, Silva AM, Irigoyen MC, Plentz RD. Functional electrical stimulation in the treatment of patients with chronic heart failure: a meta-analysis of randomized controlled trials. Eur J Cardiovasc Prev Rehabil. 2010 Jun;17(3):254-60).

21. Klompstra L, Jaarsma T, Strömberg. Exergaming in older adults: A scoping review and implementation potential for patients with heart failure. Eur J Cardiovasc Nurs. 2014 Oct;13(5):388-98. 
Table 1: Characteristics of the Exercise Training Programs provided by the LVAD implanting centers ( $\mathrm{n}=45$ ).

\begin{tabular}{|c|c|c|}
\hline Timing & $\mathrm{n}$ & $\%$ \\
\hline Phase I: in-hospital & 21 & $47 \%$ \\
\hline Phase II: Early post-discharge * & 32 & $71 \%$ \\
\hline - in-patient program: 4 weeks. & 22 & $49 \%$ \\
\hline - out-patient program: 4-12 weeks. & 13 & $29 \%$ \\
\hline - home-based program: 52 weeks. & 4 & $9 \%$ \\
\hline Phase III: Long-term maintenance program (3-52 weeks) & 11 & $24 \%$ \\
\hline Professionals involved & $\mathrm{n}$ & $\%$ \\
\hline Physiotherapist & 33 & $73 \%$ \\
\hline Psychologist & 23 & $51 \%$ \\
\hline Cardiac rehabilitation nurse & 22 & $49 \%$ \\
\hline Cardiologist specialized in rehab** & 22 & $49 \%$ \\
\hline Dietician & 21 & $47 \%$ \\
\hline Cardiologist** & 17 & $38 \%$ \\
\hline Exercise physiologist/therapist & 16 & $36 \%$ \\
\hline VAD coordinators & 13 & $29 \%$ \\
\hline Specialist in rehabilitation & 10 & $22 \%$ \\
\hline
\end{tabular}




\begin{tabular}{|c|c|c|}
\hline Cardiac surgeon & 10 & $22 \%$ \\
\hline Heart failure specialist nurse & 9 & $20 \%$ \\
\hline Specialist in internal medicine & 6 & $13 \%$ \\
\hline Occupational therapist & 6 & $13 \%$ \\
\hline Others: respiratory therapist (1) and pharmacist (1). & & \\
\hline How many VAD supported patients exercise in the same session? & $\mathrm{n}$ & $\%$ \\
\hline - 1 patient & 25 & $61 \%$ \\
\hline - 2 patients & 7 & $17 \%$ \\
\hline - 3 patients & 6 & $15 \%$ \\
\hline Tests routinely performed in VAD patients & $\mathrm{n}$ & $\%$ \\
\hline Echocardiography & 36 & $80 \%$ \\
\hline 6 minute walk test & 30 & $67 \%$ \\
\hline Quality of life & 28 & $62 \%$ \\
\hline Bicycle CPET & 24 & $53 \%$ \\
\hline Oxygen saturation & 19 & $42 \%$ \\
\hline Treadmill CPET & 6 & $13 \%$ \\
\hline Bicycle stress test without CPET & 5 & $11 \%$ \\
\hline Treadmill stress test without CPET & 1 & $2 \%$ \\
\hline
\end{tabular}




\begin{tabular}{|c|c|c|}
\hline Monitoring while exercising & $\mathrm{n}$ & $\%$ \\
\hline ECG monitor *** & 24 & $53 \%$ \\
Oxygen saturation & 3 & $7 \%$ \\
Blood pressure *** & 22 & $49 \%$ \\
\hline
\end{tabular}

most centers have several formats, therefor it add up more then 32

** most often also the person responsible for the program

*** most often combined ECG monitor and BP

VAD: Ventricular assist device, CPET: Cardio-pulmonary exercise test 
Table 2: Types of exercise training are provided to HF patients $(n=45)$

\begin{tabular}{|c|c|c|}
\hline Aerobic endurance training & 38 & $84 \%$ \\
\hline Continuous /constant load & 13 & $29 \%$ \\
\hline Interval low intensity & 21 & $47 \%$ \\
\hline Interval high intensity & 4 & $9 \%$ \\
\hline \multicolumn{3}{|l|}{ Mode of aerobic endurance training } \\
\hline Bicycle exercise training & 33 & $73 \%$ \\
\hline Walking & 28 & $62 \%$ \\
\hline Treadmill exercise training & 18 & $40 \%$ \\
\hline Other cardio fitness facilities (e.g. elliptical trainer). & 9 & $20 \%$ \\
\hline Nordic Walking & 4 & $9 \%$ \\
\hline Dynamic resistance training & 22 & $49 \%$ \\
\hline \multicolumn{3}{|l|}{ Mode of resistance training } \\
\hline Exercise equipment like resistance bands, free weights, exercise balls & 25 & $55 \%$ \\
\hline Weight machines & 18 & $40 \%$ \\
\hline Whole-body-vibration-training & 2 & $4 \%$ \\
\hline Balance training and/or training of coordinative skills & 20 & $44 \%$ \\
\hline Respiratory muscle training & 25 & $55 \%$ \\
\hline
\end{tabular}


Table 3. Reasons for not having LVAD rehab ( $\mathrm{n}=32$ centers) *

\begin{tabular}{|l|c|c|}
\hline & $n$ & $\%$ \\
\hline Patients are admitted to another cardiac rehabilitation program in the area & 14 & $44 \%$ \\
\hline Not have enough resources to open ETP to LVAD patients for: & 10 & $31 \%$ \\
Staff & 4 & $12.5 \%$ \\
Accommodation & 4 & $12.5 \%$ \\
Transportation & 5 & $16 \%$ \\
Equipment & 11 & $34 \%$ \\
\hline No reimbursement & 6 & $19 \%$ \\
\hline Lack of specialized team & 7 & $22 \%$ \\
\hline The specialist HF services already meet the needs & 7 & $22 \%$ \\
\hline Lack of evidence on safety of ETP in HF & 6 & $19 \%$ \\
\hline Lack of evidence of clinical benefits of ETP in HF & & \\
\hline
\end{tabular}

*More than one answer could be reported

LVAD: Left ventricular assist device, ETP: Exercise training program, HF: Heart failure 
Tables: Tables should be typed with double spacing, but minimising redundant space, and each should be placed on a separate sheet. Tables should be submitted, wherever possible, in a portrait, as opposed to landscape, layout. Each Table should be numbered in sequence using Arabic numerals. Tables should also have a title above and an explanatory footnote below. 